\title{
STOP, THINK SCORTCH: Re-thinking the traditional 'TORCH' screen in an era of re-emerging syphilis
}

Justin Penner ${ }^{1 \S}$, Hayley Hernstadt ${ }^{1}$, James E Burns ${ }^{2}$, Paul Randell ${ }^{3}$ Hermione Lyall ${ }^{1}$

1. Department of Paediatrics, Imperial College Healthcare Trust; London, UK

2. Centre for Clinical Research in Infectious Diseases \& Sexual Health, Institute for Global Health, University College London; London, UK

3. Department of Virology, Imperial College Healthcare Trust; London, UK

$\S$ Corresponding author: Justin J Penner, Imperial College Healthcare Trust, St Mary's Hospital, Department of Paediatrics, London, UK; justin.penner@nhs.net

Email Addresses of Authors:

JP: justin.penner@nhs.net

HH: $\underline{\text { Hayley.Hernstadt@nhs.net }}$

JEB: james.burns@ucl.ac.uk

PR: paulrandell@nhs.net

HL: hermione.lyall@nhs.net

Funding: None

Index Terms: Congenital Infections, Diagnostics

Abbreviated Title: SCORTCH: Re-Thinking the TORCH screen 


\section{Abstract}

Background: The epidemiology of congenital infections is ever changing, with a recent resurgence in syphilis infection rates seen in the UK. Identification of congenital infection is often delayed; early recognition and management of congenital infections is important. Testing modalities and investigations are often limited, leading to missed diagnostic opportunities.

Methods: The SCORTCH (syphilis, cytomegalovirus (CMV), 'other', rubella, toxoplasmosis, chickenpox, herpes simplex virus (HSV) and blood-borne viruses) acronym increases the awareness of clinicians to the increased risk of congenital syphilis, while considering other infectious aetiologies including: zika, malaria, chagas disease, parvovirus, enterovirus, HIV, hepatitis $\mathrm{B}$ and $\mathrm{C}$, and human T-lymphotropic virus 1 , in addition to the classic congenital infections recognised in the 'TORCH screen' (toxoplasmosis, 'other', rubella, CMV, HSV). The SCORTCH diagnostic approach describes common signs present in infants with congenital infection, details serological testing for mother and infant and important direct diagnostics of the infant. Direct diagnostic investigations include: radiology, ophthalmology, audiology, microbiological and PCR testing for both the infant and placental tissue, the latter also warrants histopathology.

Conclusion: The traditional 'TORCH screen' focuses on serology-specific investigations, often omits important direct diagnostic testing of the infant, and fails to consider emerging and reemerging congenital infections. In recognition of syphilis as a re-emerging pathogen and the overlapping clinical presentations of various infectious aetiologies, we advocate for a broader outlook using the SCORTCH diagnostic approach.

Keywords: congenital abnorm; infectious diseases; microbiology; neonatology; virology. 


\section{Background}

Early recognition of congenital infections remains the cornerstone of management and coordination of care, as perinatally acquired infections can be associated with significant long term sequelae if diagnosis is delayed.1 Testing practices for congenital infections vary. Differences in laboratory and direct examinations make for fragmented investigation practices.2 Furthermore, the classic 'TORCH' (toxoplasmosis, 'other', rubella, cytomegalovirus (CMV), herpes simplex virus (HSV)) serological 'screen' fails to incorporate important direct diagnostic tests and has the potential to miss key diagnoses including emerging and re-emerging congenital infections. In particular, the absence of ' $S$ ' in the 'TORCH' acronym leads to a lack of consideration for congenital syphilis, an infection with increasing incidence in the UK. 34 As presentations of congenital syphilis can vary from asymptomatic to single-system or multi-system involvement, often overlapping with the clinical presentations of other congenital infections, $\underline{56}$ we advocate for a standardised 'SCORTCH' (syphilis, CMV, 'other', rubella, toxoplasmosis, chickenpox (varicella zoster virus (VZV)), HSV and blood-borne viruses) approach. The goal of this newly titled 'SCORTCH' diagnostic toolkit is to increase primary carer education, awareness, recognition and testing of congenital infections.

\section{A Head to Toe Approach for Recognising Congenital Infections:}

Broadening the original concept of the 'TORCH screen' has been proposed, acknowledging the complexity of the diagnostic approach to congenital infections. $\underline{7-9}$ There are multiple pathogens potentially responsible for congenital infections, while overlapping clinical presentations and changing epidemiology leads to diagnostic challenges for the clinician. Overall, a high index of suspicion is required. We advocate for consideration of all possible antenatal infections given the consequences of failing to diagnose and treat perinatally acquired infections early.

An initial thorough review of the maternal antenatal history including maternal health, travel, high risk behaviours, as well as booking serology and ultrasound findings for abnormal (e.g. echogenic bowel in CMV) or absent results should be standardised. Often overlooked is the importance of obtaining placental specimens for further testing when congenital infection is possible. A comprehensive evaluation of the newborn for signs of 
congenital infection and the mother for evidence of active or past infection are required in order to institute comprehensive testing that is both accurate and timely. A complete neonatal examination reviewing central nervous, gastrointestinal, cardiovascular, lymphatic, musculoskeletal and dermatological systems is necessary when the possibility of congenital infection is raised. The newborn should have standard measurements of height, weight and head circumference recorded, with formal audiology and ophthalmological evaluations. Figure 1 outlines the initial diagnostic approach to congenital infections using the SCORTCH evaluation toolkit which emphasises consideration of multiple infectious aetiologies, particularly syphilis given the recent rise

in congenital infection. Figure 2 considers common syndromic patterns as well as an approach to asymptomatic neonates born to mothers with absent or incomplete booking bloods as they relate to individual pathogens and diagnostic modalities.

\section{SCORTCH Infection Epidemiology:}

Despite its emphasis on congenital syphilis case detection, the SCORTCH approach (Figures 1 and 2) highlights other infections previously included in the traditional ' $\mathrm{TORCH}$ screen', as well as perinatal infections and testing modalities not previously covered by the TORCH battery of serological testing.

\section{S- Syphilis}

A rise in total annual syphilis infections by 20\% from 2016 compared with 2017 exemplifies the importance of awareness and early recognition of congenital infections. Seven thousand one hundred and thirty-seven cases of syphilis were diagnosed in 2017 in the UK, a $148 \%$ increase from 2008.10 The rise in syphilis cases continues with a further 5.5\% increase reported in 2018 (7541 total cases), the largest number reported since 1949.4 Increased rates of syphilis infection have also been seen in women of childbearing age, rates reported in women 20-24 years old of 3.8/100 000.10 Recent reports by Public Health England (PHE) demonstrated increases in congenital syphilis with 21 cases diagnosed between 2010 and 2017, although reports from incident investigations suggest more widespread infection. $\underline{4}$ As clinical cases of congenital 
syphilis are on the rise, PHE has instituted a public health action plan to strengthen antenatal testing and early detection. 10

Approximately two-thirds of infants with congenital syphilis are asymptomatic at birth and thus a heightened clinical suspicion is required.11 Furthermore, a rise in atypical cases in neonates born to mothers with negative first trimester screening, and likely infected during pregnancy, require additional vigilance. $\underline{5}$ An algorithm for congenital syphilis evaluation, both for symptomatic and asymptomatic infants is outlined in figure 3 with diagnostic criteria defined in table 1. Neonatal serological evaluation should include treponemal IgM enzyme immunoassay (EIA) in conjunction with a paired quantitative nontreponemal test and quantitative Treponema pallidum particle agglutination assay (TPPA) of the infant and mother. Serological tests assessing for IgG (ie, TPPA or rapid plasma reagin) in the infant may be positive as a result of passive intrauterine transfer (to a maximum of 18 months of age) whether or not the infant is infected. A description of treponemal versus non-treponemal tests are described in table 2. Direct molecular testing (PCR) or dark field microscopy of skin lesions, nasopharyngeal aspirates and/or placenta as appropriate may assist diagnosis. In addition to standard haematological, renal and liver profiles, a neonatal lumbar puncture with CSF syphilis serology, ophthalmology review and radiographs of chest and long bones (as clinically indicated) are warranted to complete a comprehensive set of diagnostic investigations.

Table 1: Confirmatory serologic and molecular testing in congenital syphilis

\begin{tabular}{|c|c|}
\hline \multicolumn{2}{|c|}{$\begin{array}{c}\text { Suspicion of Congenital Syphilis by History/Physical exam AND/OR } \\
\text { Positive Maternal Syphilis Serology }\end{array}$} \\
\hline Confirmatory Serologic Diagnosis (12) & Confirmatory Molecular Diagnosis (12) \\
\hline $\begin{array}{l}\text { - Paired treponemal IgM EIA, quantitative RPR/VDRL and } \\
\text { TPPA on the infant* and mother } \\
\text { - Specimen with any of the following confirm diagnosis of } \\
\text { congenital syphilis: } \\
\circ \text { Positive IgM EIA in infant } \\
\circ \quad \text { Fourfold or greater difference in RPR/VDRL or TPPA } \\
\text { titre above that of the mother } \\
\circ \quad \text { Positive RPR/VDRL on CSF } \\
\circ \quad \text { Four-fold or greater increase in RPR/VDRL or TPPA } \\
\text { titre within } 3 \text { months of birth } \\
\circ \quad \text { Persistently positive treponemal tests at } 18 \text { months } \\
\text { and beyond }\end{array}$ & $\begin{array}{l}\text { - Positive syphilis PCR from exudates of suspicious } \\
\text { lesions or body fluids e.g. nasal discharge } \\
\text { - Direct demonstration of T. pallidum by dark field } \\
\text { microscopy from suspicious lesions or body fluids e.g. } \\
\text { nasal discharge }\end{array}$ \\
\hline
\end{tabular}

*infant blood, not cord blood; CSF = cerebral spinal fluid; EIA = enzyme immunoassay; $P C R$ = polymerase chain reaction; $R P R=$ rapid plasma reagin; TPPA = treponema pallidum particle agglutination assay; $V D R L=$ venereal disease research laboratory test 
Table 2: Treponemal and Non-Treponemal tests in syphilis diagnosis

\begin{tabular}{|c|c|c|}
\hline & Test & Notes \\
\hline \multirow{2}{*}{$\begin{array}{l}\text { Non-Treponemal } \\
\text { Test }\end{array}$} & VDRL & \multirow[b]{2}{*}{$\begin{array}{ll}- & \text { Positive in mother with active infection (any stage) } \\
\text { - } & \text { Gradually decreases to undetectable in mother and } \\
\text { neonate with treatment } \\
\text { - } \quad \text { May remain persistently positive at low levels after } \\
\text { adequate treatment "serofast state" with } \geq 4 \text {-fold } \\
\text { decline } \\
\text { - Titres for comparison between mother and neonate } \\
\text { important in determining need for treatment in } \\
\text { neonate }\end{array}$} \\
\hline & RPR & \\
\hline \multirow[t]{5}{*}{ Treponemal Test } & EIA/CIA/CMIA & \multirow{5}{*}{$\begin{array}{l}\text { - Remains positive lifelong even after treatment } \\
\text { - Transplacental transfer, antibodies clear in infant by } \\
\text { latest 12-18 months of age }\end{array}$} \\
\hline & TPPA & \\
\hline & TPHA & \\
\hline & MHA-TP & \\
\hline & FTA-Abs & \\
\hline
\end{tabular}

$\mathrm{CIA}=$ chemoluminescence immunoassay CMIA = chemiluminescent microplate immunoassay; $\mathrm{EIA}=$ enzyme immunoassay; FTAAbs = fluorescent treponemal antibodies - absorbed; MHA-TP = micro-haemagglutination antibodies; $\mathrm{RPR}=$ rapid plasma reagin; TPHA = treponema pallidum hemagglutination assay; TPPA = treponema pallidum particle agglutination assay; VDRL = venereal disease research laboratory test

\section{C-Cytomegalovirus}

There is increasing recognition of congenital CMV (CCMV) as an important cause of developmental delay and hearing loss. The worldwide prevalence of CCMV has been estimated at $0.5 \%-2 \%, \underline{12}$ with infected infants, regardless of symptoms at birth, at risk of developing permanent sequelae. Five to fifteen per cent of asymptomatic infants are noted to be affected later in life. 13 Largely a result of long term sequelae, the economic cost of CCMV in the UK has been estimated at $£ 732$ million. 14 This re-enforces the importance of heightened clinical awareness of congenital infections which may not be clinically apparent in the neonatal period. This parallels cases of congenital syphilis with late onset sequelae after a seemingly asymptomatic neonatal period. Figure 4 outlines a diagnostic algorithm for investigation of possible CCMV in both symptomatic and asymptomatic infants.

O- Other (Zika, Chagas, Enterovirus, Parvovirus)

The 'O' in SCORTCH has been maintained in order to highlight an evolving group of emerging and re-emerging perinatal infections. Five infectious aetiologies are referenced 
in the SCORTCH approach. Increasing global movement of people requires heightened awareness of infections acquired abroad that may have significant perinatal consequences for example malaria, trypanosomes and arboviruses. Vigilance in screening is also required as global warming and its impact on animal and insect habitats is changing the epidemiology of vector-borne diseases. Almost 1.4 million UK residents travel to zika endemic areas each year (2010-2014), of which $25 \%$ are women of childbearing age. 15 Similarly, chagas disease has recently been classified as a neglected disease in the UK following increased migration to Europe from Latin America. It has an estimated prevalence of $1.27 \%$ in at-risk residents in London alone. In view of high treatment efficacy of vertically transmitted chagas disease, the WHO recommends targeted antenatal screening which has yet to be implemented in the UK.16 The possibility of emerging and re-emerging tropical congenital infections must be surveyed closely especially given recent cases of congenital zika infections. Other flaviviruses should be considered as possible aetiologies of congenital infections as demonstrated in a recent report implicating dengue as a probable cause of congenital infection. 17 Although the risk of congenital anomalies in fetuses exposed to parvovirus has been estimated to be less than $1 \%$ in a UK study of 367 infants exposed in utero, the same study predicted fetal loss rates of approximately $10 \%$ if infected before 20 weeks gestation. 18 Fetal hydrops and/or anaemia should raise a strong suspicion of congenital parvovirus infection. Although serological testing for congenital parvovirus can be performed, highly sensitive PCR of neonatal blood should now be regarded as diagnostically superior.

Several fetal anomalies have been attributed to congenital enterovirus although the majority of cases (90\%) remain asymptomatic leading to imprecise estimates of congenital enterovirus incidence.19 Clinicians should have heightened awareness of enterovirus infection in unexplained congenital findings: severe pneumonitis, carditis, neurological manifestations, unexplained stillbirths, or with unexplained severe illness in the mother. Samples for enterovirus PCR from appropriate bodily fluids and secretions (cerebrospinal, stool, respiratory, nasopharyngeal, blood, skin) dependent on the clinical condition of the infant are indicated for diagnosis. 


\section{R-Rubella}

Measles, mumps and rubella vaccine coverage in England in 2018-2019 was 82.4\%, the lowest rate since 2011-2012.20 PHE reported 0.19 rubella infections per 100000 pregnancies between 2010 and 2016 with 0.05 cases of congenital rubella syndrome per 100000 . Since 2016, rubella has not been part of routine prenatal screening in the UK. Increased susceptibility to rubella infection in women of childbearing age leads to the possibility of increased numbers of congenital rubella cases.21 Similar to the diagnostic approach to parvovirus, direct molecular testing of an infant's secretions, blood and gestational tissue now supersedes serological testing previously advised in the ' $T O R C H$ screen'.

\section{$T$ - Toxoplasmosis}

The European Centre for Diseases Prevention and Control estimate 1.4 cases of congenital toxoplasmosis per 100000 .22 Toxoplasmosis is not routinely screened for prenatally in the UK although this is part of routine antenatal testing in other European countries. Thus, asymptomatic neonates born to mothers with known positive toxoplasmosis serology is not uncommon in the UK. This highlights toxoplasmosis as an important pathogen in both symptomatic and asymptomatic neonates requiring prompt recognition in the neonatal period, as emphasised by the SCORTCH diagnostic process. A diagnostic algorithm for toxoplasmosis serology and direct testing in symptomatic and asymptomatic neonates is included in figure 5.

\section{C-Chicken Pox (Varicella zoster virus)}

Exposure to VZV in pregnancy can cause serious consequences to non-immune pregnant women and the developing fetus. Universal varicella vaccination is not currently included in the national UK immunisation schedule despite evidence suggesting reductions of up to $85 \%$ in congenital and neonatal varicella cases in countries with universal varicella vaccination programmes. $\underline{23}$ Risks to the fetus and the neonate from maternal disease is dependent on the timing of infection in the mother. Congenital varicella syndrome is due to exposure in the first 20 weeks of pregnancy and remains rare with an estimated incidence of 1\%-2\% after maternal exposure.24 Disseminated 
neonatal infection can occur in infants born to non-immune mothers who contract VZV infection in the week before, or up to a week after delivery. 25 Given the variation of symptoms and the absence of a routine vaccination in the UK, the SCORTCH approach addresses both congenital varicella syndrome and neonatal varicella disease in its diagnostic approach. This will facilitate early diagnosis and encourage prompt implementation of prophylaxis or treatment.

\section{(i) $\boldsymbol{H}$ - Herpes Simplex Virus}

A 2017 global health report estimated the incidence of neonatal HSV disease in Europe to be 8.9 per 100000 live births.26 The British Paediatric Surveillance Unit is currently studying the incidence of neonatal HSV in the UK and is due to report it's findings in 2021. HSV is an infection that requires expedient identification and management to prevent multi-system damage and long term neurological sequelae. Seizures, skin/mucous membrane lesions, unexplained hepatitis, pneumonitis and/or or a sepsis-like clinical presentation must raise the clinician's suspicion of HSV infection. We emphasise the importance of direct diagnostic testing for HSV inclusive of: skin, eye and mucous membrane swabs and lumbar puncture for HSV PCR, with neurological imaging where applicable.

\section{(ii) H-Blood-Borne Viruses:}

\section{Human Immunodeficiency Virus (HIV), Hepatitis B (HBV), Hepatitis C (HCV), Human} T-lymphotropic virus 1 (HTLV1)

Antenatal, perinatal, and postnatal management to prevent mother-to-child transmission of blood-borne viruses is critical, and underlines the need for broad antenatal testing and response to maternal diagnosis. The 'TORCH screen' traditionally focused on diagnostic approaches for the neonate, in particular, the diagnosis of perinatal HIV. While HIV is an important focus in SCORTCH, we also advocate for broader inclusion of viral hepatitis (HBV and HCV) and HTLV-1 transmission in the diagnostic approach to congenital infections. 
The UK proportion of positive antenatal tests for HBV is $0.36 \%$ with $0.11 \%$ newly diagnosed in pregnancy, and up to $1 \%$ in certain inner city areas with higher populations with origins from endemic areas. 27 Diagnosis of maternal chronic HBV infection guides treatment of the neonate with monovalent HBV vaccine and consideration of HBV immunoglobulin as $90 \%$ of neonates infected perinatally will develop chronic infection. $\underline{27}$ Latest estimates suggest that approximately 143000 people in the UK are living with chronic HCV of which two-thirds remain undiagnosed.28 The absence of antenatal testing, particularly for HBV, should trigger urgent maternal testing. Both HBV and HCV exposure require infant follow-up and repeat testing to verify the infant's infection status. The antenatal and post-partum periods also serve as opportune times for family-focused $\mathrm{HCV}$ testing and treatment initiation as highly efficacious eradication therapy for people over 12 years is now widely available.

Similarly, maternal HIV requires rapid testing to ensure measures are undertaken perinatally and post-partum to start the mother and neonate on antiretroviral therapy and minimise mother-to-child transmission. This has been a major success in the UK over the past two decades with a steady fall in perinatal acquisition rates to $0.27 \% .29$ However, there were still 29 cases of perinatally acquired HIV in 2015, underlining the need for clinical vigilance with emphasis on urgent testing for mothers and neonates without antenatal serology results and retesting those with negative results early in pregnancy with ongoing risk factors for vertical transmission.

Lastly, in the UK it is estimated that 20 000-30 000 people are infected with HLTV-1. Early antenatal recognition, especially in those from highly endemic areas (East Asia, Middle East, Africa, South America) and those with a history of T cell lymphoma/leukaemia, may prevent up to $80 \%$ of transmissions via avoidance of breast feeding alone. $\underline{30}$

\section{Conclusions}

A broader approach to congenital infection screening is advised given the changing epidemiology of congenital infections and recent rise in syphilis diagnoses in the UK. While the traditional 'TORCH screen' focuses on ill-defined, serology-specific testing, our SCORTCH tool uses a multimodality strategy for investigations by including radiology, 
ophthalmology, audiology, microbiology and histology, as well as highly effective molecular diagnostic approaches. We advocate that medical teams use the SCORTCH approach to consider a wide range of infectious differentials given that congenital infections have overlapping clinical manifestations. 


\section{References}

1. Neu N, Duchon J, Zachariah P. TORCH infections. Clinics in perinatology. 2015;42(1):77-103, viii.

2. Hwang JS, Friedlander S, Rehan VK, Zangwill KM. Diagnosis of congenital/perinatal infections by neonatologists: a national survey. Journal of perinatology : official journal of the California Perinatal Association. 2019;39(5):690-6.

3. Mayor S. Syphilis and gonorrhoea increase sharply in England. BMJ (Clinical research ed). 2015;350:h3457.

4. Foster K SA, Hughes G, Simms I, Fifer H, Mohammed H, Webb S, Migchelsen SJ, Allen H, Ratna N, Gill O. Addressing the Increase in Syphilis in England: PHE Action Plan. London, UK: Public Health England, 2019 June 2019. Report No.

5. Furegato M, Fifer H, Mohammed H, Simms I, Vanta P, Webb S, et al. Factors associated with four atypical cases of congenital syphilis in England, 2016 to 2017: an ecological analysis. Euro surveillance : bulletin Europeen sur les maladies transmissibles = European communicable disease bulletin. 2017;22(49).

6. Simms I, Tookey PA, Goh BT, Lyall H, Evans B, Townsend CL, et al. The incidence of congenital syphilis in the United Kingdom: February 2010 to January 2015. BJOG : an international journal of obstetrics and gynaecology. 2017;124(1):72-7.

7. Ford-Jones EL, Kellner JD. "Cheap torches": an acronym for congenital and perinatal infections. The Pediatric infectious disease journal. 1995;14(7):638-40.

8. Klein JO. Current concepts of infectious diseases in the newborn infant. Advances in pediatrics. $1984 ; 31: 405-46$.

9. Dylewski J, Dobrinskikh E, Lewis L, Tonsawan P, Miyazaki M, Jat PS, et al. Differential trafficking of albumin and IgG facilitated by the neonatal Fc receptor in podocytes in vitro and in vivo. PloS one.

2019;14(2):e0209732.

10. Public Health England. Sexually Transmitted Infections (STIs): Annual Data Tables. 2018.

11. Herremans T, Kortbeek L, Notermans DW. A review of diagnostic tests for congenital syphilis in newborns. European journal of clinical microbiology \& infectious diseases : official publication of the European Society of Clinical Microbiology. 2010;29(5):495-501.

12. Kingston M, French P, Higgins S, McQuillan O, Sukthankar A, Stott C, et al. UK national guidelines on the management of syphilis 2015. International journal of STD \& AIDS. 2016;27(6):421-46.

13. Kenneson A, Cannon MJ. Review and meta-analysis of the epidemiology of congenital cytomegalovirus (CMV) infection. Reviews in medical virology. 2007;17(4):253-76.

14. Bialas KM, Swamy GK, Permar SR. Perinatal cytomegalovirus and varicella zoster virus infections: epidemiology, prevention, and treatment. Clinics in perinatology. 2015;42(1):61-75, viii.

15. Retzler J, Hex N, Bartlett C, Webb A, Wood S, Star C, et al. Economic cost of congenital CMV in the UK. Archives of disease in childhood. 2019;104(6):559-63.

16. Public Health England. UK Surveillance for Congenital Zika Syndrome 2017.

17. Requena-Mendez A, Moore DA, Subira C, Munoz J. Addressing the neglect: Chagas disease in London, UK. The Lancet Global health. 2016;4(4):e231-3.

18. Paixao ES, Teixeira MG, Costa M, Barreto ML, Rodrigues LC. Symptomatic Dengue during Pregnancy and Congenital Neurologic Malformations. Emerging infectious diseases. 2018;24(9):1748-50.

19. Miller E, Fairley CK, Cohen BJ, Seng C. Immediate and long term outcome of human parvovirus B19 infection in pregnancy. British journal of obstetrics and gynaecology. 1998;105(2):174-8. 
20. Mereaux J, Picone O, Vauloup-Fellous C, Khediri Z, Benachi A, Mandelbrot L, et al. [Enterovirus infection during pregnancy: Underestimated cause of fetal and neonatal complications?]. Gynecologie, obstetrique, fertilite \& senologie. 2017;45(4):231-7.

21. Public Health England. Childhood Vaccination Coverage Statistics - England. 2019.

22. Public Health England. Measles and Rubella Elimination UK Strategy. 2019.

23. European Centre for Disease Control. Annual Epidemiological Report: Toxoplasmosis. Stockholm: 2016.

24. Khandaker G, Marshall H, Peadon E, Zurynski Y, Burgner D, Buttery J, et al. Congenital and neonatal varicella: impact of the national varicella vaccination programme in Australia. Archives of disease in childhood. 2011;96(5):453-6.

25. Enders G, Miller E, Cradock-Watson J, Bolley I, Ridehalgh M. Consequences of varicella and herpes zoster in pregnancy: prospective study of 1739 cases. Lancet (London, England). 1994;343(8912):1548-51.

26. Amirthalingam G Bk, Ramsay M. Updated Guidelines on Post Exposure Prophylaxis (PEP) for Varicella/Shingles Public Health England 2019.

27. Looker KJ, Magaret AS, May MT, Turner KME, Vickerman P, Newman LM, et al. First estimates of the global and regional incidence of neonatal herpes infection. The Lancet Global health. 2017;5(3):e300-e9.

28. Public Health England. Hepatitis B: the Green Book. London 2019.

29. Public Health England. Hepatitis C in the UK 2019. London 2019.

30. British HIV Association. British HIV Association Guidelines for the Management of HIV in Pregnancy and Postpartum (2019 Interim Report). 2019.

31. Public Health England. 2013/2014 Standard Contract: Human T-Cell Lymphotropic Virus Type 1 \& 2 (all ages). 2013. 


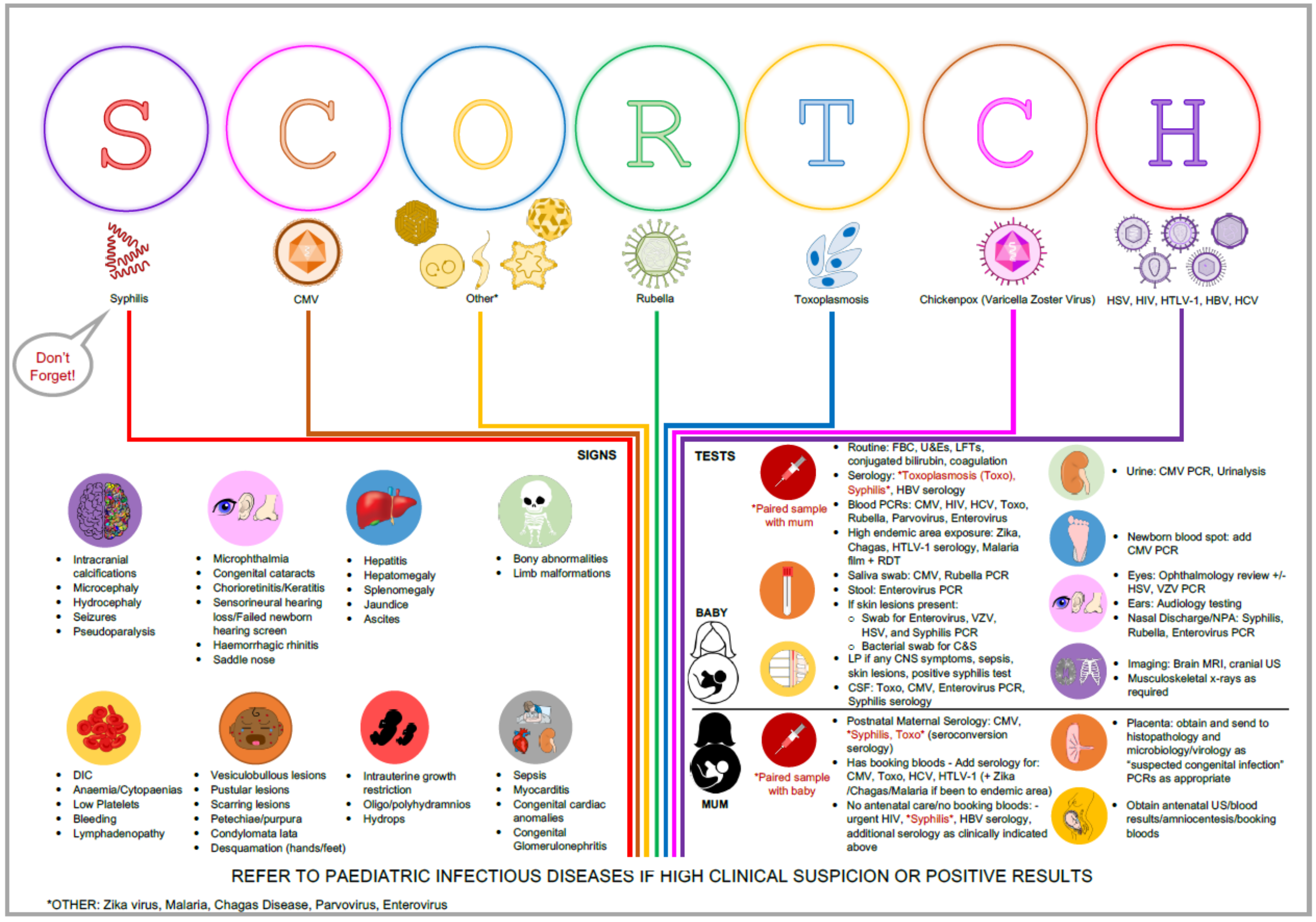

\section{Figure 1}

SCORTCH evaluation toolkit outlining a diagnostic approach for suspected congenital infections. CMV, cytomegalovirus; CNS, central nervous system; C\&S, culture and

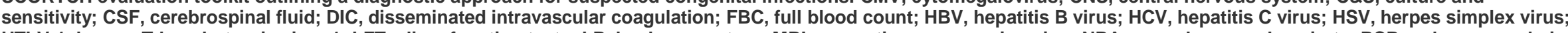

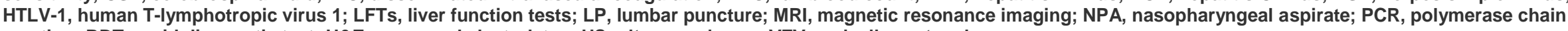
reaction; RDT, rapid diagnostic test; U\&Es, urea and electrolytes; US, ultrasound scan; VZV, varicella zoster virus. 


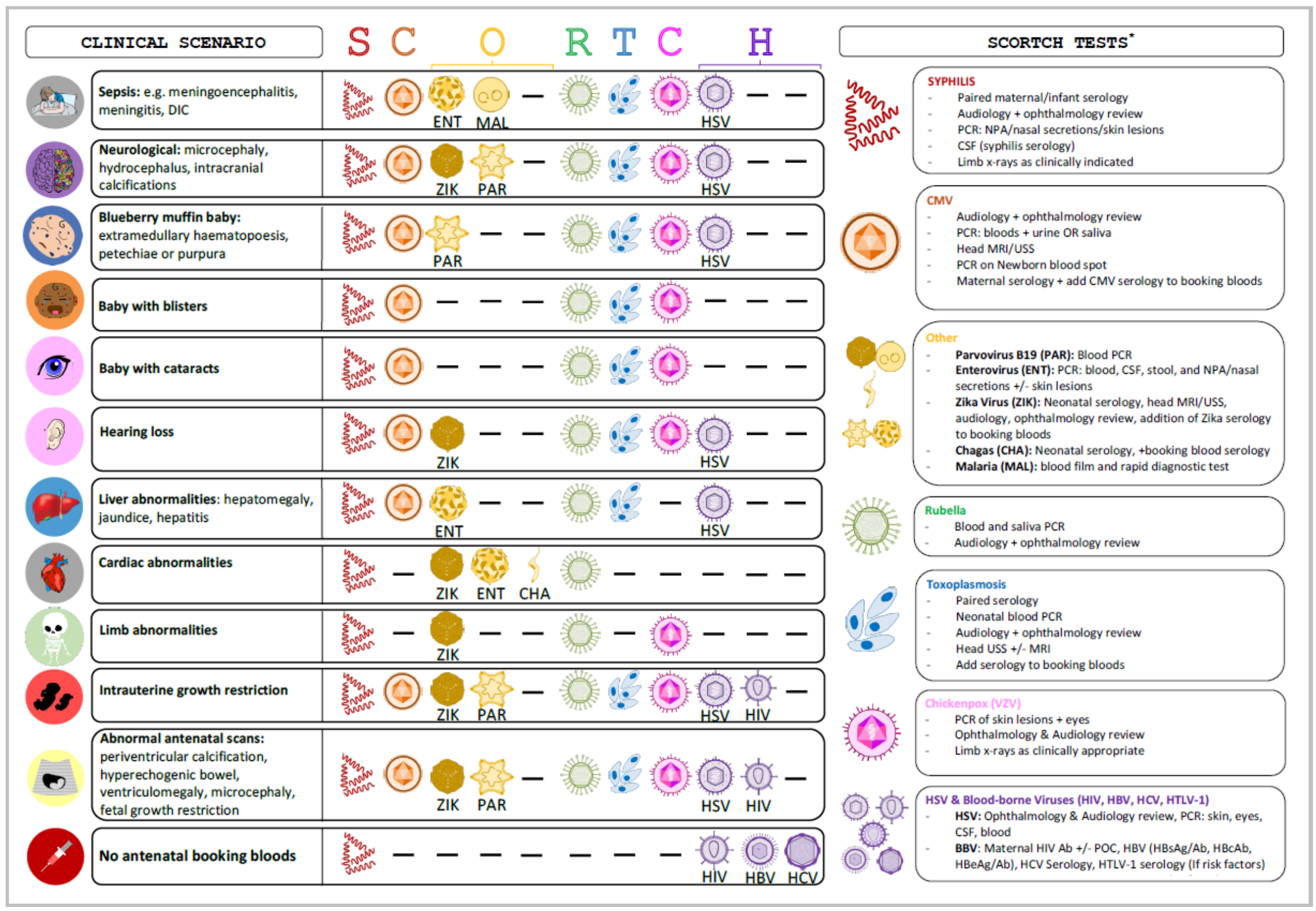

Figure 2

Syndromic application of the SCORTCH toolkit. BBV, blood-borne viruses; CMV, cytomegalovirus; C\&S, culture and sensitivity; CSF, cerebrospinal fluid; DIC, disseminated intravascular coagulation; $\mathrm{HBcAb}$, hepatitis $\mathrm{B}$ core antibody; $\mathrm{HBeAg} / \mathrm{Ab}$, hepatitis $\mathrm{B}$ e antigen/antibody; $\mathrm{HBsAg} / \mathrm{Ab}$, Hepatitis $\mathrm{B}$ surface antigen/antibody; HBV, hepatitis B virus; HCV, hepatitis C virus; HSV, herpes simplex virus; HTLV-1, human T-lymphotropic virus 1; IV, intravenous; LFTs, liver function tests; MRI, magnetic resonance imaging; NPA, nasopharyngeal aspirate; PCR, polymerase chain reaction; POC, point of care; RDT, rapid diagnostic test; SCORTCH, syphilis, CMV, 'other', rubella, toxoplasmosis, chickenpox, HSV and BBV; U\&Es, urea and electrolytes; US, ultrasound scan; VZV, varicella zoster virus. 


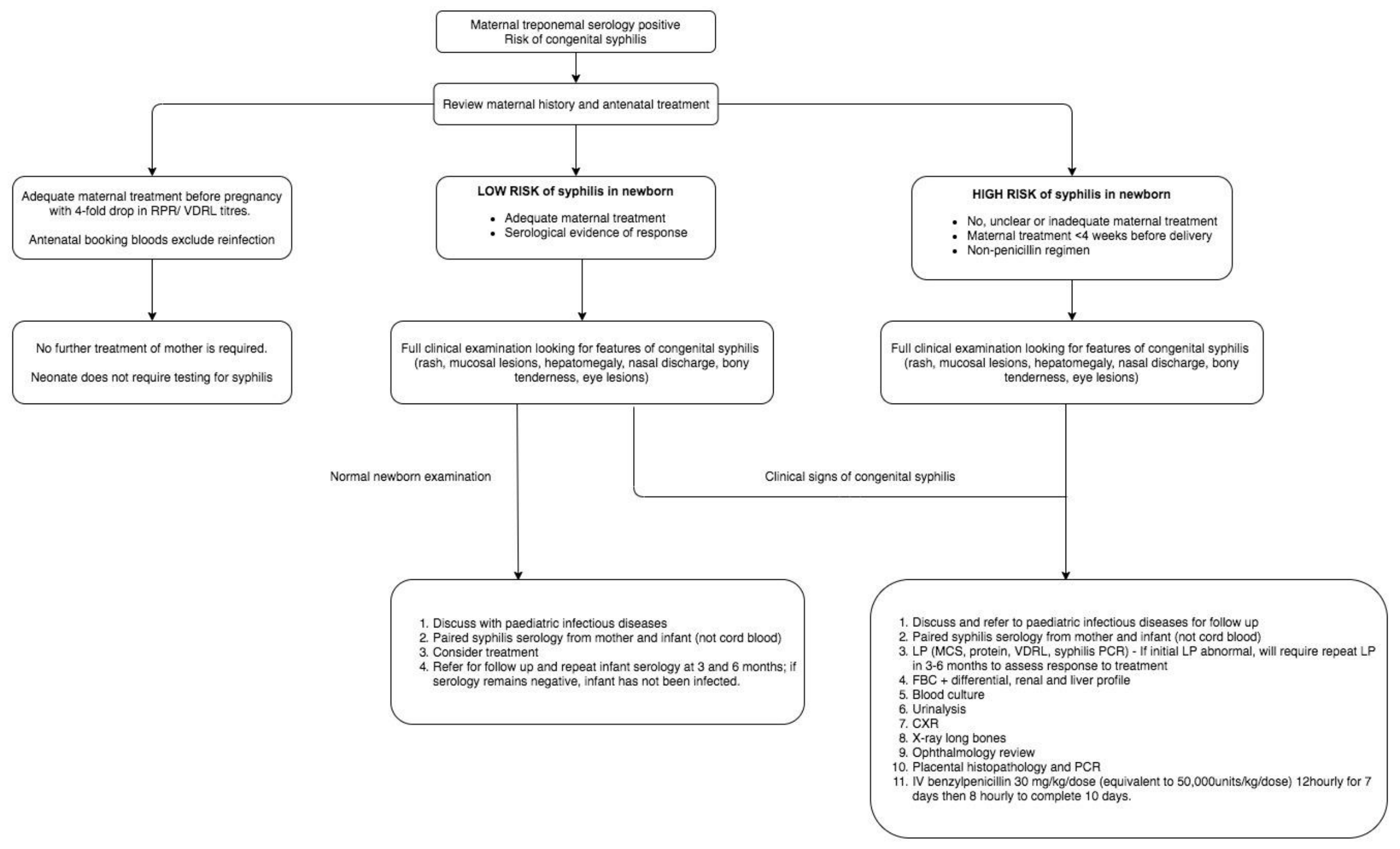

\section{Figure 3}

Diagnostic algorithm for serology, direct testing and follow-up of suspected congenital syphilis. CXR, chest X-ray; FBC, full blood count; LP, lumbar puncture; MCS, microscopy and culture and sensitivity; RPR, rapid plasma reagin; VDRL, venereal disease research laboratory test. 


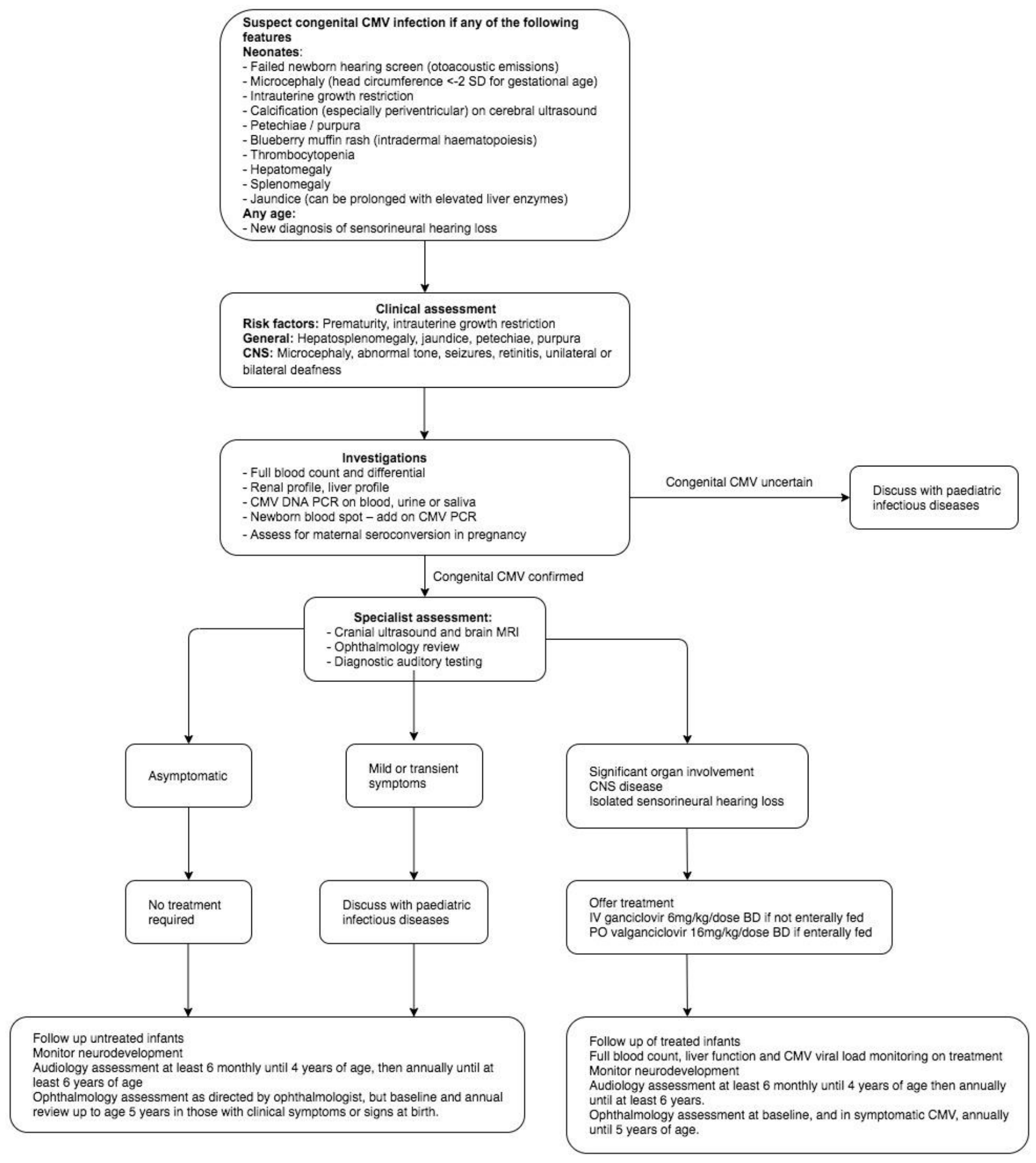

Figure 4

Diagnostic algorithm for serology, direct testing and follow-up of suspected CMV. BD, twice daily; CMV, cytomegalovirus; CNS, central nervous system; PO, per oral. 


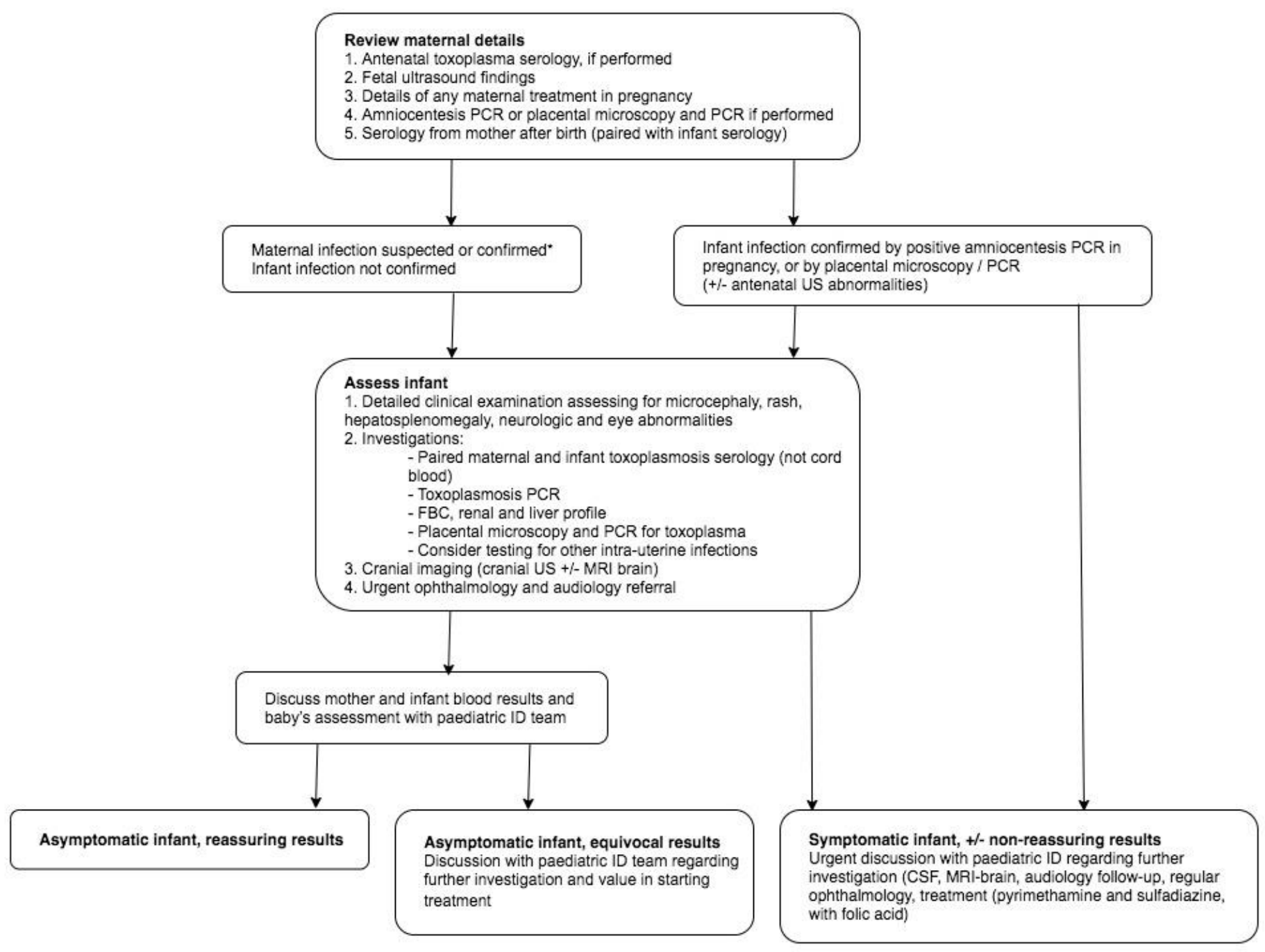

\section{Figure 5}

Diagnostic algorithm for serology, direct testing and follow-up of suspected congenital toxoplasmosis. *Risk of infection of fetus depends on trimester of maternal infection. First trimester, risk of infection $4 \%-15 \%$, likely to have severe infection if infected. Third trimester, high risk of infection $(30 \%-75 \%)$, low risk of damage if infected (4\%-17\%), usually asymptomatic at birth. CSF, cerebrospinal fluid; FBC, full blood count; ID, infectious diseases; US, ultrasound scan. 